\title{
Minkowski's footprint revisited
}

\section{Planetary nebula formation from a single sudden event? ${ }^{\star}$}

\author{
J. Alcolea ${ }^{1}$, R. Neri ${ }^{2}$, and V. Bujarrabal ${ }^{3}$ \\ 1 Observatorio Astronómico Nacional (OAN-IGN), Calle Alfonso XII 3, 28014 Madrid, Spain \\ e-mail: j.alcolea@oan.es \\ 2 Institut de Radio Astronomie Millimétrique (IRAM), 300 rue de la Piscine, 38406 St. Martin d'Hères, France \\ 3 Observatorio Astronómico Nacional (OAN-IGN), Apartado 112, 28803 Alcalá de Henares, Spain
}

Received 18 December 2006 / Accepted 12 January 2007

\section{ABSTRACT}

\begin{abstract}
Context. M 1-92 can be considered an archetype of bipolar pre-planetary nebulae. It shows a clear axial symmetry, along with the kinematics and momentum excess characteristic of this class of envelopes around post-AGB stars.

Aims. By taking advantage of the new extended configuration of the IRAM Plateau de Bure interferometer, we wanted to study the morphology and velocity field of the molecular gas better in this nebula, particularly in its central part.

Methods. We performed sub-arcsecond resolution interferometric observations of the $J=2-1$ rotational line of ${ }^{13} \mathrm{CO}$ in $\mathrm{M} 1-92$.

Results. We found that the equatorial component is a thin flat disk, which expands radially with a velocity proportional to the distance to the center. The kinetic age of this equatorial flow is very similar to that of the two lobes. The small widths and velocity dispersion in the gas forming the lobe walls confirm that the acceleration responsible for the nebular shape could not last more than 100-120 yr. Conclusions. The present kinematics of the molecular gas can be explained as the result of a single brief acceleration event, after which the nebula reached an expansion velocity field with axial symmetry. In view of the similarity to other objects, we speculate on the possibility that the whole nebula was formed as a result of a magneto-rotational explosion in a common-envelope system.
\end{abstract}

Key words. stars: circumstellar matter: jets - stars: AGB and post-AGB - stars: individual: PN M 1-92 - stars: mass-loss

\section{Introduction}

PN M 1-92, also known as Minkowski's Footprint, is an O-rich pre-planetary nebula (pPN) originally discovered by Minkowski (1946), which has been deeply observed in the optical, NIR, and radio wavelengths. The central star has a spectrum compatible with $T_{\text {eff }}=6500 \mathrm{~K}$ and $\log g=0.5$, although at short wavelengths it shows an excess that can be fitted with the presence of a secondary star of $T_{\text {eff }}=18000 \mathrm{~K}$ and $\log g=5.0$ (Arrieta et al. 2005). The distance to M 1-92 has been estimated as $2.5 \mathrm{kpc}$, adopting a nominal luminosity of $10^{4} L_{\odot}$ for a post-AGB star (Cohen \& Kuhi 1977). The nebula has a size of $11^{\prime \prime}$ in the axial direction and $6^{\prime \prime}$ in the transversal one. It consists of a two-lobe reflection nebula, divided by a dense equatorial component. The two lobes define a clear axis of symmetry, oriented at a position angle (PA) of $311^{\circ}$ (measured from north to east). This axis is inclined in respect to the plane of the sky by about $35^{\circ}$, with the northwest lobe pointing to us. Optical spectroscopy shows the presence of wide absorption components, revealing the existence of (bipolar) flows of ionized gas close to the star, with expansion velocities up to $750 \mathrm{~km} \mathrm{~s}^{-1}$ (Arrieta et al. 2005). Line emission in $\mathrm{H} \alpha$ and in atomic forbidden lines (O I, O III, N II, S II) is detected from the middle of the two lobes (Bujarrabal et al. 1998b),

* Based on observations carried out with the IRAM Plateau de Bure Interferometer. IRAM is supported by INSU/CNRS (France), MPG (Germany) and IGN (Spain). probably tracing the location of shocks propagating along these jets (see also Solf 1994). Bujarrabal et al. (1998b) estimated that the mass of the ionized gas in the nebula is only about $10^{-3} M_{\odot}$, and Arrieta et al. (2005) concluded that the present ionized mass loss is very low, $3 \times 10^{-13} M_{\odot} \mathrm{yr}^{-1}$.

Because of the early stage of the central star in its post-AGB evolution, almost all the nebular material is still in the form of molecular gas. This component has been studied in detail by Bujarrabal et al. (1998a) by means of interferometric observations of the $J=1-0$ and $J=2-1$ rotational lines of ${ }^{13} \mathrm{CO}$, obtaining a resolution of $\sim 1{ }^{\prime \prime} 0$ at $220 \mathrm{GHz}$ (in the $J=2-1$ line). The molecular gas is located in the equatorial disk and in the walls of the two lobes, which are practically empty. From those maps, the total mass estimated for the molecular component is $0.9 M_{\odot}, 0.2 M_{\odot}$ of which is located in the disk and $0.35 M_{\odot}$ in the walls of each lobe. The excitation was found to be very low across the nebula, from 10 to $15 \mathrm{~K}$, and the densities ranging from $5 \times 10^{4}$ to $3 \times 10^{5} \mathrm{~cm}^{-3}$, the warmer and densest parts located close to the equator. The velocity field of the gas was also investigated using a spectral resolution of $3.25 \mathrm{~km} \mathrm{~s}^{-1}$. These authors found that the kinematics consists in a low radial expansion component of $8 \mathrm{~km} \mathrm{~s}^{-1}$, which dominates in the equatorial region, plus an axial component that increases linearly with the distance to the equatorial plane, reaching an expansion velocity of $70 \mathrm{~km} \mathrm{~s}^{-1}$ at the two nebular tips. Such a Hubble-like velocity law was interpreted as the result of a sudden interaction 
that lasted much less than the kinetic age of the axial outflow $(\sim 1000 \mathrm{yr})$. In addition, an axial momentum of $22 M_{\odot} \mathrm{km} \mathrm{s}^{-1}$ was measured in the nebula. This value is much larger that what can be provided by radiation pressure on dust grains on the expected acceleration lime (about $100 \mathrm{yr}$ or less). In fact these two properties, the presence of Hubble-like axial acceleration, and the momentum excess are very common among pPNe (Bujarrabal et al. 2001).

In spite of all these observational efforts, very little is known about the mechanism that accelerated and stretched the nebula along its symmetry axis, as well as about what is powering the present bipolar outflow seen in ionized gas. (Note that the jet seen in the optical does not have enough energy or momentum to explain the present kinematics, so we may be in the presence of two different processes.) This is partially due to the limited spatial resolution of the molecular line observation, which cannot reveal the structure of the lobe walls or the shape and size of the equatorial component. Making use of the new, improved resolution of the Plateau de Bure interferometer, in this letter we report on new sub-arcsecond resolution observations of M 1-92 in the ${ }^{13} \mathrm{CO} J=2-1$ line.

\section{New Plateau de Bure observations}

These new observations of M 1-92 were conducted with the new extended configurations $6 \mathrm{Aq} \& 6 \mathrm{~Bq}$ of the IRAM Plateau de Bure mm-wave interferometer. The observations were performed on January 24 and February 26, 2006 (configurations A and $\mathrm{B}$, respectively), under excellent to good weather conditions. Using the $1.3 \mathrm{~mm}$ receiver, we observed the $J=2-1$ rotational line of ${ }^{13} \mathrm{CO}$, adopting a rest frequency of $230399 \mathrm{MHz}$. The $J=1-0$ lines of ${ }^{13} \mathrm{CO}$ and $\mathrm{C}^{18} \mathrm{O}$ were simultaneously observed with the $3 \mathrm{~mm}$ receiver, but their results will be discussed elsewhere. The correlator was set up to cover the whole $J=2-1$ line of ${ }^{13} \mathrm{CO}$ with an original spectral resolution of $156.25 \mathrm{kHz}$, i.e. $0.20 \mathrm{~km} \mathrm{~s}^{-1}$ at $230.4 \mathrm{GHz}$; however, to ease the comparison with previous maps of the nebula, we re-sampled the channels to the same velocity resolution used before. To improve the reliability of the phase calibration, water vapor corrections based on the results of the $22 \mathrm{GHz}$ radiometers installed on the antennas were applied. Final phase calibration was performed from the observations of the quasars J1923+210 and J2023+336 (inter-spaced every $20 \mathrm{~min}$ ), which were also used as secondary amplitude calibrators. Observations of the radio-sources 3C 273, 3C 345, and MWC 349 were performed to fix the absolute flux scale and to calibrate the gain across the band.

After the data set was calibrated, maps were produced and CLEANed. The maps from the new data showed a significant fraction of lost flux, $\sim 50 \%$ on average, when compared with previous observations. These losses are smaller, only $\sim 30 \%$, for velocities that are both extreme and close to the systemic one. In contrast, for intermediate expansion velocities, 5-20 $\mathrm{km} \mathrm{s}^{-1}$, the missing flux fraction is as high as $\sim 2 / 3$. In order to recover this lost emission and produce maps with both high-contrast and spatial resolution, we merged our new data with those from our 1995-1997 observations (Bujarrabal et al. 1998a), using robust weighting. The resulting clean-beam has an HPBW of $0{ }^{\prime} 51 \times 00^{\prime} 35$, with the major axis oriented at PA $=36^{\circ}$. This clean beam was later used in the CLEANing procedure following the Clark method. Comparing the recovered flux in this data set with that from our previous observations both with Pico de Veleta and Plateau de Bure, we conclude that all spectra are compatible within the uncertainties and that no flux is lost in the maps. The final rms for the adopted spectral resolution of
$3.25 \mathrm{~km} \mathrm{~s}^{-1}$ is $4.5 \mathrm{mJy}$ beam $^{-1}$, and the conversion from these flux units into Rayleigh-Jeans equivalent brightness temperature $T_{\mathrm{B}}$ is $142 \mathrm{~K}_{\text {per Jy beam }}{ }^{-1}$. In addition we produced maps using just the visibilities from the most extended configuration (6Aq) and a spectral resolution of $0.5 \mathrm{~km} \mathrm{~s}^{-1}$, to better investigate the structure and velocities in the equatorial component. The resulting clean-beam has an HPBW of $0.46 \times 0.26$ at PA of $25^{\circ}$, the final rms is $15 \mathrm{mJy}^{-1} \mathrm{bem}^{-1}$, and the conversion from these flux units into $T_{\mathrm{B}}$ is $208 \mathrm{~K}$ per Jy beam ${ }^{-1}$.

\section{Observational results}

The maps are shown in Fig. 1 and the corresponding position vs. velocity diagram along the symmetry axis is displayed in Fig. 2 (left panel). The observations clearly show the structure of the nebula, with its two empty lobes divided by the equatorial disk, and the axial Hubble-like velocity field. The lobe walls are very thin, with a de-convolved $F W H P$ of $0.25\left(9 \times 10^{15} \mathrm{~cm}\right)$. The inner limits of these walls are much better defined than the outer boundary, where some weak diffuse emission is detected, especially at low velocities. The velocity dispersion in the walls must also be very small, since we can hardly detect it with a $3.25 \mathrm{~km} \mathrm{~s}^{-1}$ resolution. At the nebular tips, these widths and velocity dispersion are larger, about 0.9 and $5 \mathrm{~km} \mathrm{~s}^{-1}$, respectively. All these figures are typically $10-15 \%$ of the distance to the center and of the expansion velocity at the corresponding points. This puts a strong upper limit on the duration of the forces responsible for accelerating the nebula along the symmetry axis; we confirm that the process could not last more than about one tenth of the kinetic age of the flow: i.e. $\$ 100-120 \mathrm{yr}$ (see later).

At the center of the nebula we find that the equatorial structure is not compatible with our previous model. We do not see the signatures of a disk expanding at a constant radial velocity (see e.g. Chiu et al. 2006). This is much better displayed with the results from the 6Aq configuration (Fig. 2, center and right panels). In the axial velocity cut, we find that this structure also shows a constant velocity gradient, but in the opposite sense. In the maps, we see that the emission at each velocity traces a strip parallel to the symmetry axis. All these features can only be fitted with a disk in which the radial expansion is not constant but proportional to the distance to the center: i.e. we also find a Hubble-like velocity field in the equatorial plane, a totally unexpected result! Note that the maximum in the maps arises from the center of this disk and at the systemic velocity, in total disagreement with the expectations for an equatorial undisturbed remnant of an AGB envelope. From the extent of the emission at the systemic velocity, we derive that the diameter of the disk is $1^{\prime \prime} .4\left(5 \times 10^{16} \mathrm{~cm}\right)$ and the height at half power is $\lesssim 0 \prime^{\prime} .2$ $\left(7 \times 10^{15} \mathrm{~cm}\right)$. At the resolution achieved in the maps, we do not find a central cavity or signatures of gas rotation.

For the axial and equatorial outflows, we find a projected velocity gradient of 7.6 and $12 \mathrm{~km} \mathrm{~s}^{-1}$ per arc-second, respectively. Assuming the distance and inclination with respect to the plane of the sky given in Sect. 1, these figures translate into kinetic ages, $t_{\text {kin }}$, of 1060 and $1370 \mathrm{yr}$, respectively. However, since the two flows are perpendicular to each other, these kinetic ages have an opposite dependence with the adopted inclination angle. In particular, changing this angle from $35^{\circ}$ to $38.5^{\circ}$ (i.e. only by $10 \%$ and within the uncertainties in our previous modeling), both ages become the same, $1200 \mathrm{yr}$. This inclination is still compatible the one derived by Solf (1994) of $33^{\circ} \pm 5^{\circ}$, and the resulting coeval scenario is much more plausible than having one ejection along the equatorial plane $300 \mathrm{yr}$ before the the axial flow is launched. If this hypothesis is correct, the whole 


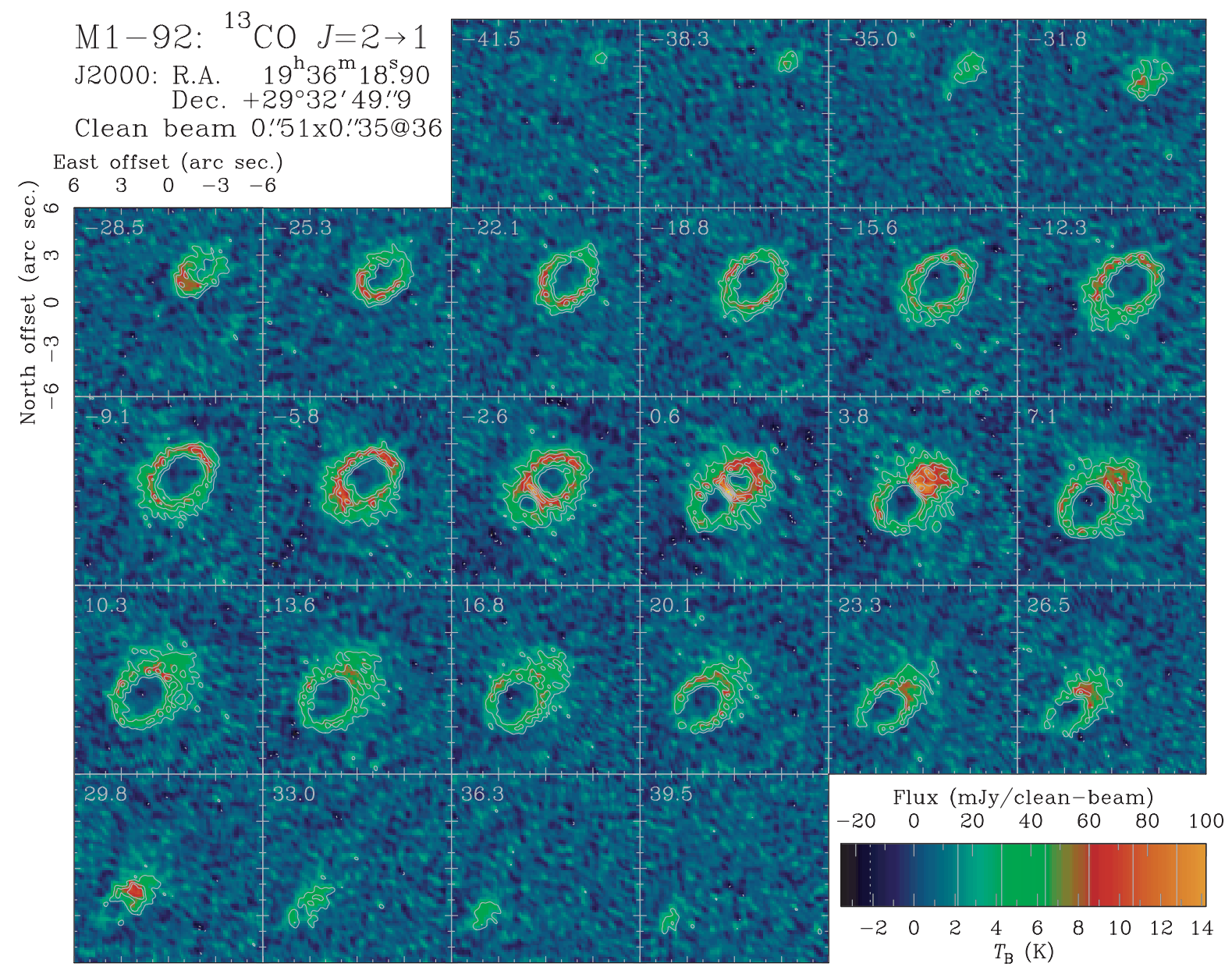

Fig. 1. The $3.25 \mathrm{~km} \mathrm{~s}^{-1}$ resolution channel maps of the intensity of ${ }^{13} \mathrm{CO} J=2-1$ in $\mathrm{M} 1-92$ from the new+old Plateau de Bure data (see text). The LSR velocity in $\mathrm{km} \mathrm{s}^{-1}$ for the center of each channel is indicated in the upper left corner. Drawn contours are -15 (dashed), and 15 to 90 by $10 \mathrm{mJy}_{\text {beam }}{ }^{-1}$. The assumed coordinates for the reference position are indicated in the top left corner of the plot.

CO nebula would share the same Hubble-like velocity field: the velocity would just be proportional to the distance, $\boldsymbol{V}=\boldsymbol{R} / t_{\mathrm{kin}}$, and we can simply use the velocity along the line of sight to derive the gas distribution in this direction. In this case the position vs. velocity digram shown in Fig. 2 would represent the spatial structure of M 1-92, if we forget our finite resolution in velocity and space, by simply translating the angular distance and velocities into length units using the distance to the target and the derived velocity gradient. Note that even the location of the weak diffuse emission seen along the equatorial plane at velocities around $10-15 \mathrm{~km} \mathrm{~s}^{-1}$ (Fig. 2 left) seems consistent with the halo detected in the maps in Fig. 1.

\section{The origin of the pPN M 1-92}

The origin of bipolar outflows in pPNe is not yet understood. Current scenarios involve the presence of accretion on the central star or on the companion in the case of binary systems through a rotating-disk, magneto-centrifugal launching, and a variety of collimating agents (Balick \& Frank 2002). The question of whether close binary systems are mandatory for producing axissymmetric nebulae is also a long-standing issue (Bujarrabal et al. 2000). Of course explaining the formation of a nebula involving coeval axial and equatorial Hubble-like flows becomes even more difficult.

However, M 1-92 is not the only object in which complex structures resulting from a single sudden event have been found.
The brightest luminous blue variable, $\eta$ Car, is surrounded by a cloud of gas and dust, whose central and densest part is known as the "Homunculus". This nebula, with an estimated mass $\sim 1 M_{\odot}$, consists of a two-lobe structure divided by a thin equatorial torus. Morse et al. (2001) performed a proper motion study in this nebula by comparing HST images taken at three different epochs and concluded that the "Homunculus" also shows a single Hubble law and was formed in less that $10 \mathrm{yr}$ during the great eruption in the 1840's. These measurements are only done in the plane of the sky (neither the position nor the velocities are derived along the line of sight, as in our case), so they do not depend on the orientation of the nebula or on the distance. Although $\eta$ Car and the central system in M 1-92 are very different, the striking similarity found in the kinematics and shape of these two nebulae strongly suggests that they may share a common origin. Matt et al. (2006) have developed a model for $\eta$ Car in which the differential rotation between the stellar nucleus and its envelope can drive a magneto-rotational explosion. In this model, as a result of the sudden release of the magnetic energy when it overcomes the weight of the upper layers, a bipolar jet is launched but an equatorial flow is driven too. These authors suggest that this mechanism would also explain the shapes of bipolar pPNe like IRAS 17106-3046 (Matt \& Balick 2004). Recently Nordhaus \& Blackman (2006) conclude that for this mechanism to operate, how the differential rotation is sustained against the increasing magnetic force can be explained by the angular momentum transferred to the stellar envelope by a 

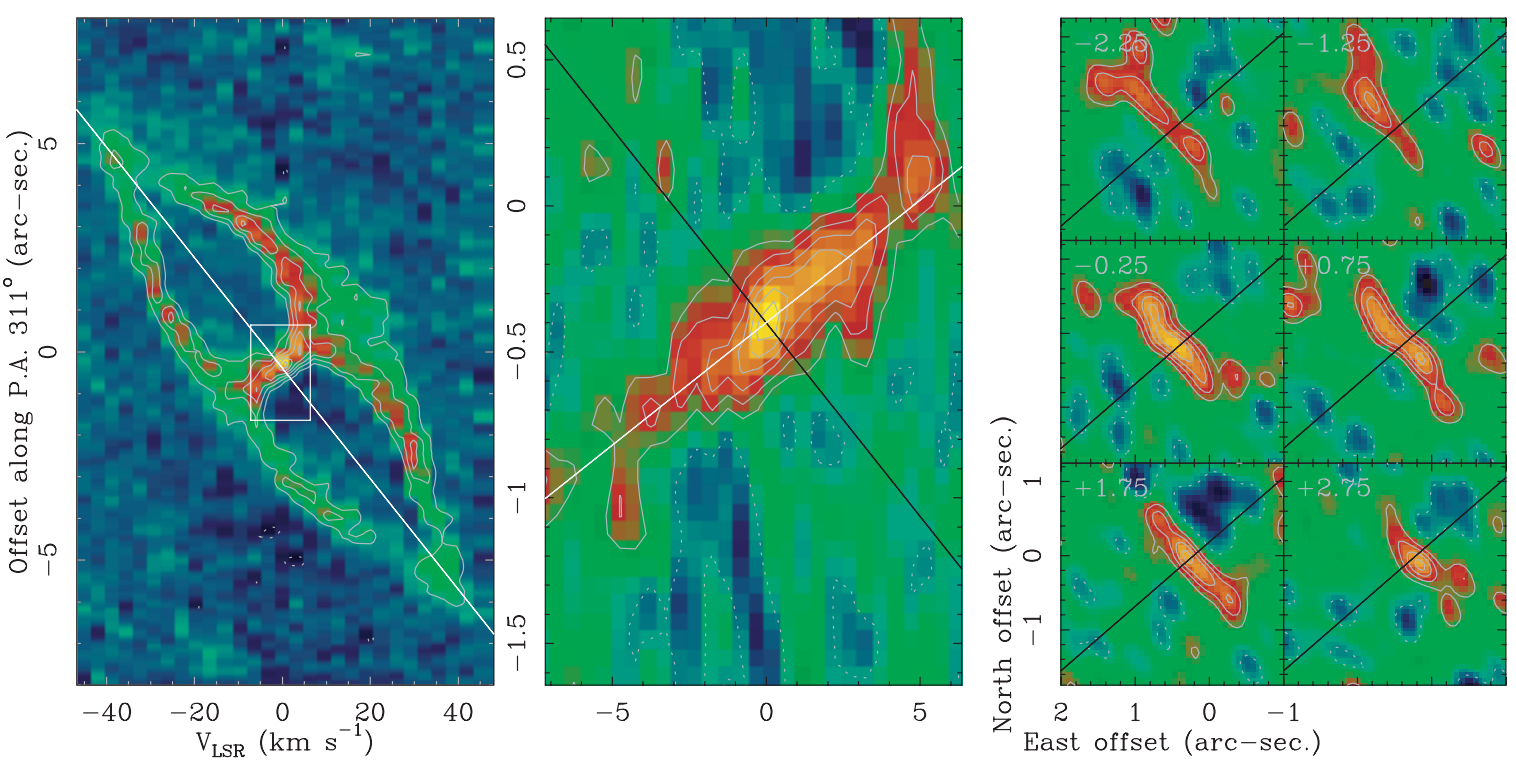

Fig. 2. Left: position vs. velocity diagram for a cut along the symmetry axis of the nebula (PA $311^{\circ}$ ). Contours are as in Fig 1 . The straight line shows the velocity gradient found along this direction. Center: the same for the central region of the nebula (the rectangle shown on the left) from the maps obtained only with $6 \mathrm{Aq}$ data and for a spectral resolution of $0.5 \mathrm{~km} \mathrm{~s}^{-1}$. Contours are as before. The white/black straight lines show the gradient of the equatorial/extended components of the nebula. Right: selected channel velocity maps of the center of the nebula, obtained just from the $6 \mathrm{Aq}$ data with $0.5 \mathrm{~km} \mathrm{~s}^{-1}$ resolution. Contours are as before. The straight line delineate the axial direction. (See also Fig. 1.)

low-mass companion during a common envelope phase. If this is also the case for M 1-92, we should conclude that this pPNe is largely formed by gas directly expelled from the stellar envelope and not a former AGB circumstellar envelope accelerated by the interaction with some post-AGB flow. Otherwise it would be very difficult to explain how the present gas could show such an accurate ballistic movement.

Although M 1-92 is so far the only case in which we can argue that the central disk was formed at the same time as the lobes, there are other pPNe in which radial acceleration has been found along the equator in addition to the typical bipolar flows. In CRL 2688 Cox et al. (2000) found in the CO maps up to 7 different bipolar outflows, three along the axis of symmetry (D to $\mathrm{F}$ in their naming) and four more (A to C and G) that could be running in different directions in the equatorial plane. In all these jets the velocity increases with the distance to the center. The authors do not comment on whether a Hubble law also holds here, but from Fig. 3 in that paper we conclude that a constant velocity gradient is consistent with the data. However, it is very difficult to see whether these equatorial flows have similar kinetic ages (so as to compare them with those for the axial jets). For the axial flows, this Hubble-law velocity field has been recently confirmed by Ueta et al. (2006), by means of proper motion measurements using HST NICMOS images. Another interesting case is CRL 618. Here Sánchez Contreras et al. (2004) observed ${ }^{12} \mathrm{CO}$, and when fitting their results with a numerical model, they found that an increasing velocity was also necessary for the central dense core. This model neither assumed an exact Hubble law field nor constrained this kinematics to a thin equatorial disk, but note that the fitting was not very precise in this region because of the limited spatial resolution of the observations. (In these two cases, the interaction of the post-AGB flows with the old AGB shell is clearly detected.) Finally, Frosty Leo presents a dense equatorial disk/torus that expands at higher velocities than those typical in AGB envelopes. Castro-Carrizo et al. (2005) conclude that such a high velocity was probably due to interactions between the AGB and post-AGB flows in the a plane perpendicular to the axial direction. However, in view of our current finding, we may also speculate with the hypothesis of an equatorial acceleration in this source, too. Note, however, that M 1-92 is one the very few $\mathrm{pPN}$ in which the molecular gas has been studied at such high spatial and spectral resolution. Therefore it would very premature to conclude whether the mechanism proposed here for explaining the kinematics of M 1-92 should also apply to other pPNe. We must wait until high-resolution images of the molecular gas in more sources are available, but we think that this possibility should be explored in the context of understanding the issues of $\mathrm{pPNe}$ and $\mathrm{PNe}$ formation and shaping.

Acknowledgements. J.A. and V.B. acknowledge their partial support from the Spanish Ministry of Education and Science under projects AYA2003-7584 and ESP2003-04957. Data reduction and plots have been performed using the Gildas software package.

\section{References}

Arrieta, A., Torres-Peimbert, S., \& Georgiev, L. 2005, ApJ, 623, 252

Balick, B., \& Frank, A. 2002, ARA\&A, 40, 439

Bujarrabal, V., Alcolea, J., \& Neri, R. 1998a, ApJ, 504, 915

Bujarrabal, V., Alcolea, J., Sahai, R., Zamorano, J., \& Zijlstra, A. A. 1998b, A\&A, 331, 361

Bujarrabal, V., García-Segura, G., Morris, M., Soker, N., \& Terzian, Y. 2000, in Asymmetrical Planetary Nebulae II: From Origins to Microstructures, ed. J. H. Kastner, N. Soker, \& S. Rappaport, ASP Conf. Ser., 199, 201

Bujarrabal, V., Castro-Carrizo, A., Alcolea, J., \& Sánchez Contreras, C. 2001, A\&A, 377, 868

Castro-Carrizo, A., Bujarrabal, V., Sánchez Contreras, C., Sahai, R., \& Alcolea, J. 2005, A\&A, 431, 979

Chiu, P.-J., Hoang, C.-T., Dinh-V-Trung, et al. 2006, ApJ, 645, 605

Cohen, M., \& Kuhi, L. V. 1977, ApJ, 213, 79

Cox, P., Lucas, R., Huggins, P. J., et al. 2000, A\&A, 353, L25

Matt, S., \& Balick, B. 2004, ApJ, 615, 921

Matt, S., Frank, A., \& Blackman, E. G. 2006, ApJ, 647, L45

Minkowski, R. 1946, PASP, 58, 305

Morse, J. A., Kellogg, J. R., Bally, J., et al. 2001, ApJ, 548, L207

Nordhaus, J., \& Blackman, E. G. 2006, MNRAS, 370, 2004

Sánchez Contreras, C., Bujarrabal, V., Castro-Carrizo, A., Alcolea, J., \& Sargent, A. 2004, ApJ, 617, 1142

Solf, J. 1994, A\&A, 282, 567

Ueta, T., Murakawa, K., \& Meixner, M. 2006, ApJ, 641, 1113 